\title{
INTRINSIC CHARACTERIZATION OF POLYNOMIAL TRANSFORMATIONS BETWEEN VECTOR SPACES OVER A FIELD OF CHARACTERISTIC ZERO ${ }^{1}$
}

\author{
BY G. R. BLAKLEY ${ }^{2}$
}

Communicated by P. T. Bateman, December 19, 1967

1. Introduction. Examples. A complex valued function $u$ of a complex argument is a polynomial function $u(z)=\mathfrak{a} z^{3}+\mathfrak{b} z^{2}+\mathfrak{c} z+\mathfrak{d}$ of degree at most three if and only if $u$ satisfies the inhomogeneous inclusion-exclusion identity of degree three

$$
\begin{aligned}
& u(\beta+\gamma+\delta \delta)-u(\beta-\gamma+\delta \delta)-u(\beta+\gamma-\delta \delta)+u(\beta-\gamma-\delta \delta) \\
& =\delta(u(\beta+\gamma+\delta)-u(\beta-\gamma+\delta)-u(\beta+\gamma-\delta)+u(\beta-\gamma-\delta)),
\end{aligned}
$$

for all complex numbers $\beta, \gamma, \delta, \delta$. The function $u(z)=z+1$ is a polynomial function of degree at most three. Suppose a real valued function $t$ of two real arguments is Euler homogeneous of degree three. Then $t$ is a cubic form $t(x, y)=e x^{8}+\mathfrak{f} x^{2} y+\mathfrak{g} x y^{2}+\mathfrak{h} y^{3}$ if and only if either $t$ satisfies the heterogeneous inclusion-exclusion identity of degree three

$$
\begin{aligned}
(t(\beta+\gamma+\delta \delta)-t(-\beta+\gamma+\delta \delta)-t(\beta-\gamma+\delta \delta)-t(\beta+\gamma-\delta \delta)) / 24 \\
=\delta(t(\beta+\gamma+\delta)-t(-\beta+\gamma+\delta)-t(\beta-\gamma+\delta)-t(\beta+\gamma-\delta)) / 24,
\end{aligned}
$$

for all ordered pairs $\beta, \gamma, \delta$ of real numbers, all real numbers $\delta$, or $t$ satisfies the homogeneous inclusion-exclusion identity of degree three

$$
\begin{aligned}
(t(\mathfrak{b} \beta+\mathfrak{g} \gamma & +\delta \delta)-t(-\mathfrak{b} \beta+\mathfrak{g} \gamma+\mathfrak{d} \delta)-t(\mathfrak{b} \beta-\mathfrak{g} \gamma+\mathfrak{b} \delta)-t(\mathfrak{b} \beta+\mathfrak{g} \gamma-\mathfrak{d} \delta)) / 24 \\
& =\mathfrak{b} \mathfrak{g} b(t(\beta+\gamma+\delta)-t(-\beta+\gamma+\delta)-t(\beta-\gamma+\delta)-t(\beta+\gamma-\delta)) / 24
\end{aligned}
$$

for all ordered pairs $\beta, \gamma, \delta$ of real numbers, all real numbers $\mathfrak{b}, \mathfrak{g}, \mathfrak{b}$. The annihilator map $t(x, y)=0$ is a cubic form.

This paper gives the general characterization of polynomial transformations between vector spaces over a field of characteristic zero. The characterization, a generalization of A. M. Gleason's [3] and H. Röhrl's [9] recent treatment of quadratic forms, is in terms of inclusion-exclusion [4, pp. 8-10] identities. It is analogous to the characterization of a linear map $v$ by means of the linearity identity $v(\mathfrak{a} \alpha+\mathfrak{b} \beta)=\mathfrak{a} v \alpha+\mathfrak{b} v \beta$. Constant, linear and affine maps do not fit

1 Presented, in part, to the Society on September 1, 1967.

2 Supported, in part, by NSF Grant GP 6106 and, in part, by NSF Grant GP 7446. 
neatly into the inclusion-exclusion identity theory. As far as it is concerned there is a disparity between the straight (degrees zero and one) and the curved (degrees two and higher).

2. Euler homogeneous maps. Polynomial transformations. Let $V$ (with zero vector $\theta$ ) and $W$ (with zero vector $\omega$ ) be vector spaces over a field $\Omega$ of characteristic zero. Let $J$ be the set $W^{v}$ of functions (maps, transformations) with domain $V$, codomain $W$. Let $r$ be a nonnegative integer. A map $s \in J$ is Euler homogeneous of degree $r$ if for each $\mathfrak{a} \in \Omega$, each $\alpha \in V$ it is true that $s \mathfrak{\alpha} \alpha=\mathfrak{a}^{r} s \alpha$. A map $t \in J$ is a homogeneous polynomial transformation of degree $r$ if there is an $r$ linear map $m: V \times V \times \cdots \times V \rightarrow W$ such that for each $\alpha \in V$ it is true that $\operatorname{ta}=\boldsymbol{m}(\alpha, \alpha, \cdots, \alpha)$. Homogeneous polynomial transformation of degree $r$ are Euler homogeneous maps of degree $r$. Let $r$ be a nonnegative integer. A map $u \in J$ is a polynomial transformation of degree at most $r$ if there is an $r$-affine map a: $V \times V \times \cdots \times V \rightarrow W$ such that for each $\alpha \in V$ it is true that $u \alpha=a(\alpha, \alpha, \cdots, \alpha)$. Let $t \in J$. If $t$ is a homogeneous polynomial transformation of degree $r$ then $t$ is a polynomial transformation of degree at most $r^{*}$ for each integer $r^{*} \geqq r$. Let $u \in J$. If $u$ is an affine map then $u$ is a polynomial transformation of degree at most $r$ for each positive integer $r$. If $u$ is a polynomial transformation of degree at most $r$ then $\boldsymbol{u}$ is a polynomial transformation of degree at most $r^{*}$ for each integer $r^{*} \geqq r$.

ThEOREM. Let $u \in J$. Let $r$ be a nonnegative integer. Fix any integer $r^{*} \geqq r$. Suppose that $u$ is a polynomial transformation of degree at most $r^{*}$. Suppose that $\mathrm{u}$ is an Euler homogeneous map of degree $r$. Then $\mathrm{u}$ is a homogeneous polynomial transformation of degree $r$.

Theorem. Let $r$ be a nonnegative integer. A map $u \in J$ is a polynomial transformation of degree at most $r$ if and only if there is a homogeneous rth degree polynomial transformation $u^{*}: V \oplus \Omega \rightarrow W$ such that for each $\alpha \in V$ it is true that $u \alpha=u^{*}(\alpha, 1)$.

GLeason's Lemma. $t \in J$ is a homogeneous polynomial transformation of degree two if and only if both of the following assertions hold:

$$
(t(\gamma+\delta \delta)-t(-\gamma+\delta \delta)) / 4=\delta(t(\gamma+\delta)-t(-\gamma+\delta)) / 4
$$

for each $b \in \Omega$, each $\gamma, \delta \in V$.

(ii) Either $\operatorname{ta} \alpha=\mathfrak{a}^{2} t \alpha$ for each $\mathfrak{a} \in K$, or else $t \theta=\omega$.

3. Inclusion-exclusion identities. Now fix an integer $p \geqq 2$. Let $L=\{1,2, \cdots, p\}$. If $S$ is a finite set let $n(S)$ be the number elements of $S$. Thus $n(\phi)=0$ and $n(L)=p$. Let 


$$
\begin{aligned}
M & =\{A \subset L \mid 2 n(A)<p\} \cup\left\{A^{*} \subset L \mid 2 n\left(A^{*}\right)=p \text { and } 1 \in A^{*}\right\}, \\
N & =\{A \subset L \mid 1 \notin A\} .
\end{aligned}
$$

Let $2^{L}$ be the power set of $L$. The set $D^{L}$ consists of all lists [7, p. 43] of $p$ elements of the set $D$. Define a function $r: 2^{L} \rightarrow\{1,-1\}^{L}$ by setting $r[B](j)=-1$ if $j \in B, r[B](k)=1$ if $k \in B$ for each $B C L$. Now let

$$
\begin{aligned}
g(p, t, \alpha) & =\sum_{A \in M}(-1)^{n(A)} t\left(\sum_{j \in L} r[A](j) \alpha(j)\right) / p ! 2^{p-1} \\
g^{*}(p, u, \beta) & =\sum_{B \in N}(-1)^{n(A)} u\left(\sum_{j \in L} r[B](j) \beta(j)\right) .
\end{aligned}
$$

If $\mathfrak{a} \in \Omega^{L}$ and $\alpha \in V^{L}$ define the pointwise product $\mathfrak{a} \alpha \in V^{L}$ by setting $(\mathfrak{a} \alpha)(i)=\mathfrak{a}(i) \alpha(i)$ for each $i \in L$. Let

$$
Y=\left\{\mathfrak{b} \in \Re^{L} \mid \mathfrak{b}(j)=1 \text { for each } j \in L \sim\{p\}\right\} .
$$

A map $u \in J$ satisfies the inhomogeneous inclusion-exclusion identity of degree $p$ if $\mathfrak{g}^{*}(p, \mathfrak{u}, \mathfrak{b} \beta)=\mathfrak{b}(p) \mathfrak{g}^{*}(p, \mathfrak{u}, \beta)$ for each $\mathfrak{b} \in Y$, each $\beta \in V^{L}$. A map $t \in J$ satisfies the heterogeneous inclusion-exclusion identity of degree $p$ if $\mathfrak{g}(p, t, \mathfrak{a} \alpha)=\mathfrak{a}(p) \mathfrak{g}(p, t, \alpha)$ for each $\mathfrak{a} \in Y$, each $\alpha \in V^{L}$. A map $t \in J$ satisfies the homogeneous inclusion-exclusion identity of degree $p$ if

$$
\mathscr{S}(p, t, \mathfrak{a} \alpha)=\mathfrak{a}(1) \mathfrak{a}(2) \cdots \mathfrak{a}(p) \mathscr{S}(p, t, \alpha)
$$

for each $\mathfrak{a} \in \Omega^{L}$, each $\alpha \in V^{L}$.

TheOREM. Let $u \in J$. Suppose $u$ is an affine map. Then $u$ satisfies the inhomogeneous inclusion-exclusion identity of degree $p^{*}$ for each integer $p^{*} \geqq 2$.

TheOREM. Let $t \in J$. Suppose that $t$ is an Euler homogeneous map of degree 1. Suppose that there is an integer $p^{*} \geqq 2$ such that $t$ satisfies the inhomogeneous inclusion-exclusion identity of degree $p^{*}$. Then $t$ is a linear map.

Theorem. Let $t \in J$. Suppose that $t$ is an Euler homogeneous map of degree $p$. Then $t$ satisfies the homogeneous inclusion-exclusion identity of degree $p$ if and only if $t$ satisfies the heterogeneous inclusion-exclusion identity of degree $p$.

To each $t \in J$ there corresponds a map $m[p, t]: V \times V \times \cdots$ $\times V \rightarrow W$ defined by setting, for $\beta \in V^{L}$, 


$$
m[p, t](\beta(1), \beta(2), \cdots, \beta(p))=g(p, t, \beta) .
$$

Theorem. Let $t \in J$. Suppose $t$ is an Euler homogeneous map of degree $p$. Suppose $t$ satisfies the homogeneous inclusion-exclusion identity of degree $p$. Then $m[p, t]$ is a symmetric multilinear map. Moreover for each $\lambda \in V$ it is true that $t \lambda=m[p, t](\lambda, \lambda, \cdots, \lambda)$.

This is a von Neumann-Jordan theorem [6] whose proof uses Gleason's lemma.

4. Intrinsic characterizations. Recall the blanket hypothesis of this paper, that $p \geqq 2$.

Homogeneous Characterization Theorem. $t \in J$ is a homogeneous polynomial transformation of degree $p$ if and only if $t$ is Euler homogeneous of degree $p$ and $t$ satisfies one of the following identities:

(i) The heterogeneous inclusion-exclusion identity of degree $p$.

(ii) The homogeneous inclusion-exclusion identity of degree $p$.

(iii) The inhomogeneous inclusion-exclusion identity of degree $p$.

(iv) The inhomogeneous inclusion-exclusion identity of some degree $p^{*} \geqq p$.

(v) The inhomogeneous inclusion-exclusion identity of each degree $p^{*} \geqq p$.

Inhomogeneous Characterization Theorem. $u \in J$ is $a$ polynomial transformation of degree at most $p$ if and only if $u$ satisfies the inhomogeneous inclusion-exclusion identity of degree $p$.

There are three ideas behind the proofs [1] of all these results. To prove that polynomial transformations satisfy inclusion-exclusion identities go back to the definitions in terms of multilinear and multiaffine maps. Write out the combinations and verify the identities. To prove that a degree $p$ Euler homogeneous map $t \in J$, which satisfies that the homogeneous degree $p$ inclusion-exclusion identity is in fact of the form

$$
t \alpha=m[p, t](\alpha, \alpha, \cdots, \alpha)
$$

where $\boldsymbol{m}[p, t]$ is a symmetric multilinear map from $V \times V \times \cdots \times V$ to $W$, employ Gleason's Lemma and a combinatorial argument to show that $m[p, t]$ is symmetric bilinear in any two of its arguments for a fixed setting of the other $p-2$. To get the general theory from the homogeneous theory without having to adapt all the proofs of the foregoing results to multiaffine maps employ the definition of arbitrary polynomial transformations from $V$ to $W$ in terms of homogeneous polynomial transformations from $V \oplus \Omega$ to $W$. Some interesting technical lemmas are the following. 
Lemma. Suppose $u \in J$ is a polynomial transformation of degree at most $p$. Define $t: V \oplus \Re \rightarrow W$ by setting

$$
\begin{aligned}
& t(\beta, 0)=\sum_{j=0}^{p}\left(\begin{array}{l}
p \\
j
\end{array}\right)(-1)^{j} \mathfrak{u}((p-2 j) \beta) / p ! 2^{p} \\
& t(\beta, \mathfrak{b})=\mathfrak{b}^{p} \mathfrak{u}((1 / \mathfrak{b}) \beta)
\end{aligned}
$$

for each $\beta \in V$, each nonzero $\mathfrak{b} \in \Omega$. Then $t$ is a homogeneous polynomial transformation of degree $p$.

LEMmA. Suppose $t \in J$ satisfies the homogeneous inclusion-exclusion identity of degree $p$. Then for each $\beta \in V$ it is true that $(-1)^{p} t(-\beta)=t \beta$.

5. Differential calculus. Let $V$ and $W$ be Banach spaces. The Frechet derivative of $t$ with respect to $\alpha \in V$ at $\beta$ is the limit

$$
\langle t: \alpha\rangle(\beta)=\lim (t(\beta+\mathfrak{h} \alpha)-t(\beta)) / \mathfrak{h}
$$

as $\mathfrak{h} \rightarrow 0$. The mixed $p$ th order Frechet derivative $[8$, p. 169] of $t \in J$ with respect to the vectors on the list $\gamma \in V^{L}$ at the vector $\beta \in V$ is defined as

$$
\begin{aligned}
\langle t: \gamma(1), \gamma(2), \cdots, \gamma(p-1) & , \gamma(p)\rangle(\beta) \\
& =\langle\langle t: \gamma(1), \gamma(2), \cdots, \gamma(p-1)\rangle: \gamma(p)\rangle(\beta) .
\end{aligned}
$$

The vector $\langle t: \delta, \delta, \cdots, \delta, \delta\rangle(\beta)=\left\langle t: \delta^{p}\right\rangle(\beta)$ is the pure $p$ th Frechet derivative of $t$ with respect to $\delta \in V$ at $\beta$.

EULER's TheOREM. If $t \in J$ is an Euler homogeneous map of degree $p$ then for each $x \in L$, each $\alpha \in V$ it is true that

$$
(p-x) !\left\langle t: \alpha^{x}\right\rangle(\alpha)=p ! t \alpha .
$$

The Archimedean Mean Value Theorem. Suppose $t \in J$ is $a$ homogeneous polynomial transformation of degree $p+1$. Suppose $\alpha^{*} \in V^{L \cup\{p+1\}}$, that $\alpha^{*}(p+1)=\eta$, and that $\alpha$ is the restriction of $\alpha^{*}$ to $L$. Then

$$
(p+1) ! 2^{p} g\left(p+1, t, \alpha^{*}\right)
$$

$$
=2 \sum_{A \in M}(-1)^{n(A)}\langle t: \eta\rangle\left(\sum_{j \in L} r[A](j) \alpha(j)\right) .
$$

Suppose that $p=1$, so that $t$ is quadratic. If $W, V$ and $\Omega$ are the real numbers then the graph of $t$ is a parabola through the origin. The theorem then implies that $t(\alpha+1)-t(\alpha-1)=2 t^{\prime}(\alpha)$. This last fact, in its geometric form, was known to the Greeks [5, p. 234] before Archimedes. An induction based on this theorem leads to 
Dixon's Theorem on Differences and Derivatives. Suppose $V$ and $W$ are Banach spaces and that $t \in J$ is a homogeneous polynomial transformation of degree $p$. Then for each $\alpha \in V^{L}$ it is true that

$$
p ! g(p, t, \alpha)=\langle t: \alpha(1), \alpha(2), \cdots, \alpha(p-1)\rangle(\alpha(p)) .
$$

This observation [2] of R. D. Dixon puts the foregoing theory into a new light. The theory was developed as a purely combinatorial exercise. But he has given very different proofs, valid in Banach spaces, of several of the results above.

Added in proof. S. Kurepa's papers (Glasnik Mat.-Fiz. Astronom. Ser. II Društvo Mat. Fiz. Hrvatske 19(1964), 23-26 and 20(1965), 79-92) parallel [3] and [9]. Polynomial transformations between affine $[7$, p. 420$]$ spaces $A, B$ over a field $\Omega$ of characteristic zero have an intrinsic characterization. $s \in B^{A}$ is a quadratic polynomial transformation if and only if

$$
s(\mathfrak{a} \alpha+\mathfrak{b} \beta)=s(\mathfrak{b} \alpha+\mathfrak{a} \beta)+(\mathfrak{a}-\mathfrak{b}) s(\alpha)+(\mathfrak{b}-\mathfrak{a}) s(\beta)
$$

for each $\alpha, \beta \in A$ each $\mathfrak{a}, \mathfrak{b} \in \mathfrak{\Omega}$ such that $\mathfrak{a}+\mathfrak{b}=1$. The characterization of a cubic polynomial transformation $s \in B^{A}$, in a symmetric form which can be given an intrinsic affine meaning, is that

$$
\begin{aligned}
{[\mathfrak{s}(\mathfrak{a} \alpha+\mathfrak{b} \beta)-s(\mathfrak{a} \beta+\mathfrak{b} \alpha)]-} & {[s(\mathfrak{a}(\Omega \alpha)+\mathfrak{b}(\Omega \beta))-s(\mathfrak{a}(\Omega \beta)+\mathfrak{b}(\Omega \alpha))] } \\
= & {[(\mathfrak{a s}(\alpha)+\mathfrak{b s}(\beta))-(\mathfrak{a s}(\beta)+\mathfrak{b s}(\alpha))] } \\
& -[\mathfrak{a s}(\Omega \alpha)+\mathfrak{b s}(\Omega \beta)-(\mathfrak{a s}(\Omega \beta)+\mathfrak{b s}(\Omega \alpha))]
\end{aligned}
$$

for each $\alpha, \beta \in A$, each translation $\Omega$ of $A$, each $\mathfrak{a}, \mathfrak{b} \in \Re$ such that $\mathfrak{a}+\mathfrak{b}=1$. The affine inclusion-exclusion identity characterizations of higher degree transformations will appear in [1].

\section{REFERENCES}

1. G. R. Blakley, Theory of nonlinear transformations. I: Polynomial transformations between vector spaces over a field of characteristic zero, (in preparation).

2. R. D. Dixon, Private communication.

3. A. M. Gleason, The definition of a quadratic form, Amer. Math. Monthly 73 (1966), 1049-1056.

4. M. Hall, Combinatorial theory, Blaisdell, Waltham, Mass., 1967.

5. T. L. Heath, The works of Archimedes, Dover, New York, 1957.

6. P. Jordan and J. von Neumann, On inner products in linear metric spaces, Ann. of Math. 36 (1935), 719-723.

7. S. Mac Lane and G. Birkoff, Algebra, Macmillan, New York, 1967.

8. H. K. Nickerson, D. C. Spencer and N. E. Steenrod, Advanced calculus, Van Nostrand, Princeton, N. J., 1959.

9. H. Röhrl, Zur Definition quadratischer Formen, Math. Z. 98 (1967), 42-45.

UNIVERSITY OF ILLINOIS AND

State University of New York at Buffalo 